\title{
Concevoir des robots pour développer la créativité des élèves ?
}

John Didier, Haute école pédagogique du canton de Vaud (Suisse), UER Arts et technologie et Florence Quinche, HEP Vaud, UER Médias et TIC

\section{Introduction}

L'introduction de la robotique à l'école primaire et secondaire a débuté depuis une dizaine d'années dans plusieurs établissements scolaires de Suisse romande (Quinche et Didier). Le robot en tant qu'objet technique (Simondon) induit des représentations issues encore majoritairement du monde industriel et liées aux activités de production, au rendement et à la répétition, ce qui place trop souvent la robotique dans un univers où l'individu et sa créativité ont peu de place. Pourtant, dans un contexte de formation et d'apprentissage, le robot offre des possibilités pour travailler l'interdisciplinarité et la créativité. C'est ce que nous souhaitons montrer dans cet article. Depuis longtemps, la robotique est enseignée dans les écoles à l'aide de marches à suivre, ou sur le modèle des mathématiques sous la forme de problèmes fermés ${ }^{1}$ laissant très peu de marge à la créativité des élèves.

Pour nous dissocier de ces manières d'enseigner la robotique, essentiellement focalisées sur la production et les résultats au détriment des apprentissages des élèves, nous privilégions une approche par projet (Didier et Quinche). Ceci nous permet de nous situer dans des exigences du nouveau plan d'études (PER), qui demande que la créativité soit travaillée avec les élèves en tant que compétence transversale (donc dans différentes disciplines). Nos objectifs sont de travailler au sein d'un même projet : l'apprentissage de la démarche scientifique, la modélisation (et le travail sur la construction de représentations) et des objectifs communicationnels (utiliser le robot comme objet-signe dans une pièce de théâtre). Notre pari étant qu'en réalisant des projets qui mettent en œuvre différentes disciplines (Activités créatrices et manuelles (ACM), sciences, technologie, français, Médias et TIC), on favorise la créativité des élèves. La robotique en tant qu'outil pédagogique (Quinche et Didier) offre, en outre, des possibilités d'apprendre à agir et à décider dans des espaces de contraintes qui vont nécessiter des solutions innovantes et adaptées au contexte. Comment comprenons-nous le terme de robotique ? Sous plusieurs aspects : design, construction du robot et programmation, mais aussi socialisation du robot (objet signifiant dans la production théâtrale). L'aspect de construction matérielle du robot étant aussi important que sa programmation.

Nous proposons cette démarche de formation dans une école pédagogique (HEP Vaud) destinée aux futurs enseignant-e-s. Ces étudiant-e-s seront de futurs enseignant-e-s généralistes 
du primaire. Ces cours s'inscrivent dans le cadre d'un module interdisciplinaire (Activités créatrices et manuelles, Médias et technologies) de troisième année qui vise l'intégration de la technologie au sein d'autres disciplines, mais aussi le développement des capacités transversales (pensée créatrice, réflexivité, collaboration). Aucune connaissance préalable de la robotique ou de l'informatique n'est demandée aux étudiant-e-s.

Dans ce projet, l'étudiant-e en formation apprend à concevoir des situations d'enseignement-apprentissage destinées à des élèves, mais il doit au préalable tester l'ensemble des activités prévues et réaliser un projet collectif avec d'autres étudiant-e-s. Le travail est réalisé en groupe de deux à quatre personnes.

\section{Cadre théorique}

\subsection{Conception, réalisation et socialisation du robot}

L'introduction de la robotique dans le cadre de la formation des futurs enseignant-e-s demande de dépasser un enseignement purement transmissif pour privilégier un apprentissage par expérimentations et par questionnements. En effet, se familiariser avec ce champ complexe demande des compétences pratiques et pas simplement des connaissances théoriques. Pour permettre aux étudiant-e-s de s'approprier différentes manières de travailler les capacités transversales par le biais de la robotique, nous mettons en œuvre le modèle ConceptionRéalisation-Socialisation ${ }^{2}$ (Didier et Leuba). L'intérêt de ce modèle consiste à privilégier une analyse fonctionnelle de l'objet pour identifier les besoins des futurs usagers. Dès le début de son projet, l'étudiant-e devra ainsi réfléchir à la socialisation du robot qui sera réalisé. Dans notre exemple, il devra imaginer le rôle du robot dans la pièce de théâtre et le sens véhiculé pour le spectateur de la pièce. Ainsi, nous quittons rapidement un apprentissage par essaiserreurs pour définir la fonctionnalité du robot, son introduction dans la séquence d'enseignement et son apport au niveau des apprentissages. Le modèle ConceptionRéalisation-Socialisation (C-R-S) permet aux étudiant-e-s de définir dès le début du projet les enjeux de la mission ou le cahier des charges du robot. Il nécessite donc d'analyser la mission et les différentes contraintes qui vont paramétrer l'action du robot. Ce modèle « C-R-S » induit différentes productions sémiotiques. Les étudiant-e-s devront trouver des moyens de modéliser : dessins, croquis, plans, schémas, maquettes, etc. Ils apprennent ainsi à partager et à communiquer leurs idées avec les autres membres du groupe.

Ceci facilite l'apprentissage de la programmation et de la conception d'un robot ou d'une partie du robot. Le travail par groupe renforce l'apprentissage de la conception collective, essentielle dans la plupart des métiers techniques et culturels. Les compétences 
communicationnelles s'enrichissent par les interactions verbales (de type informatif, mais aussi négociation et argumentation nécessaire dans la prise de décision), mais également des traces écrites, graphiques ou photographiques. Il est également demandé aux étudiant-e-s de garder des traces des différentes étapes de la conception (journal d'atelier, notes, photographies, séquences de film, etc.), afin d'expliciter le processus de création et ses différentes étapes (développement de la réflexivité).

\subsection{Conception et genèse de l'objet}

Le robot est introduit dans ce module en tant qu'objet technique (Simondon) qui nous renseigne sur notre propre rapport aux machines et à leur fonctionnement dans notre quotidien, mais également comme objet potentiellement inclus dans des productions culturelles et donc porteur de sens et d'interprétations. Le robot se caractérise en tant qu'objet interdisciplinaire favorisant un enseignement par projet qui convoque plusieurs disciplines scolaires : les activités créatrices et manuelles, les TIC, le français, les mathématiques, les sciences.

Les objets réalisés dans la scolarité obligatoire se caractérisent en tant qu'espace d'analyse favorisant la compréhension des objets techniques (Deforge). Ceux-ci se situent au carrefour de différents champs disciplinaires tout en se différenciant d'un objet purement scientifique (Simondon). En explorant l'objet technique nous induisons une approche pragmatique de l'analyse de l'objet et de la genèse qui le constitue.

La difficulté consiste à accéder à la genèse de l'objet technique, car chaque objet se veut le résultat d'une évolution et d'une individualité qui lui est spécifique. De ce fait, comprendre un robot et son évolution nécessite de le situer en rapport à l'évolution des automates, de la mécanique, de l'électronique et de l'informatique. La conception d'un objet donne accès à la distinction des phases de production et donne lieu à l'analyse des contraintes spécifiques de celles-ci en fonction de l'utilisateur et du contexte. De ce fait, la spécificité de cet artefact est en lien avec la culture technique. La culture technique comprend aussi bien la culture des métiers que la culture d'entreprise (Terssac) et permet de comprendre l'historicité des techniques. En appréhendant le robot à travers son histoire et son apport dans la culture, nous privilégions une approche technologique compatible avec une approche en sciences humaines (Deforges ; Lamard et Lequin ; Simondon). Concevoir et programmer un robot nous amène à mieux comprendre l'évolution des techniques et les choix sociaux qui ont permis son évolution. Lorsque nous demandons aux étudiant-e-s de concevoir et de réaliser un ascenseur à l'aide du robot WeDo, nous les amenons à se questionner et à comprendre le fonctionnement ainsi que l'évolution des objets du quotidien. 
Construire un robot ou l'intégrer dans une histoire nécessite d'aller au-delà d'une simple utilisation fondée sur l'essai erreur. Concevoir un programme ou une partie d'un robot à réaliser induit une compréhension de ses fonctions et de sa socialisation (la façon dont l'objet sera utilisé et perçu par ses utilisateurs, ou encore compris par son public). L'activité de conception en amont de sa construction et de sa programmation doit prendre en compte les contraintes de réalisation et les contraintes liées aux interactions avec l'usager. Dans cette phase de conception, les étudiant-e-s sont amené-e-s à dessiner des représentations du robot et à imaginer des solutions techniques pour sa construction. Il s'agit là d'un apprentissage de la modélisation. Dès lors, les résultantes liées à la conception du robot nous amènent à mieux cerner l'évolution et les phases spécifiques apparaissant lors du processus de production (ceci englobe les phases de programmation).

\subsection{Conception et créativité}

Pour comprendre la démarche de conception, nous privilégions l'introduction de missions concrètes dans lesquelles le robot doit être construit et programmé. Mais comment mieux saisir les mécanismes cognitifs sollicités lors de la conception? Notre première entrée en matière se penche plus attentivement sur le processus créatif. Pour Joy Paul Guilford et Ellis Torrance, la créativité est perçue en termes de capacité induite qui peut être mesurée (Lubart). Nous définissons la créativité en tant que « capacité à produire une idée exprimable sous une forme observable ou à réaliser une production qui soit à la fois novatrice et inattendue, adaptée à la situation et (dans certains cas) considérée comme ayant une certaine utilité ou de valeur » (Bonnardel 21). Nous utilisons dans ce module l'approche multivariée issue de la psychologie de la créativité (Lubart) qui distingue des facteurs : cognitifs (intelligence, connaissance), conatifs (personnalité des concepteurs de l'objet, motivation), émotionnels et environnementaux. Ces facteurs influent sur le potentiel créatif. Il est intéressant de relier les activités de conception avec celles impliquant de la créativité, notamment dans l'expression d'une idée, ou dans la réalisation d'une production ou d'un processus en fonction d'un but. Dans ces deux cas de figure, il y a bien recherche d'une adaptation à la situation ou au contexte (Bonnardel). Selon Mihaly Csikszentmihalyi, les productions créatives sont évaluées au sein d'un système social et c'est par rapport à ce dernier qu'est définie la nouveauté. La créativité est, en effet, fréquemment reliée à la nouveauté alors que cet aspect est rarement mis en avant dans les définitions de la conception où l'on distingue plutôt les activités routinières et nonroutinières (Mayer, voir Bonnardel). Mais comment favoriser des processus de conception incitant à la production d'objets innovants et adaptés ?

\subsection{Quelles situations-problèmes pour favoriser la créativité ?}


L'introduction de différents défis (en termes d'objectifs et de contraintes), qui doivent être résolus par le robot, développe chez le concepteur/la conceptrice la mise en œuvre de processus créatifs. De cette manière, il/elle se voit confronté-e à toute une série de problèmes à résoudre à l'aide de solutions qui devront être inventées et adaptées au contexte (environnement, matériel à disposition, moyens, temps, etc.). Des défis de plus en plus complexes sont proposés par les formateurs au cours des différents séminaires. La construction et la programmation d'un petit ascenseur va progressivement laisser la place à des scénarios plus élaborés dans lesquels des personnages vont être introduits et associés à des petits scénarios dans lesquels sont réintroduits ce type de constructions. Les situations-problèmes qui posent des difficultés de conception et favorisent la créativité, peuvent ressembler à un ensemble de contraintes, qui ne sont pas exprimées en termes techniques, mais en termes de fonction ou de signification de l'objet : par exemple on demande aux étudiants de réaliser un objet hybride reprenant à la fois les caractéristiques d'un animal existant et celles d'une machine. En d'autres termes, on leur demande de réaliser une chimère. Cette situationproblème n'induit pas de réponse préétablie, et il n'y a pas de réponse juste ou fausse. S'y insère la liberté de choix des concepteurs, qui s'apparente à celle de l'artiste ou de l'auteur-e qui se donne un thème et des contraintes pour la réalisation de son ouvre. On pourra considérer l'objet comme réussi, ou comme fonctionnant dans le contexte souhaité si les objectifs de socialisation sont atteints : que voulait-on produire comme effet avec cet objet ? Etonner, faire rire ? Générer un questionnement sur le mouvement animal (à la manière des bioroboticiens) ? L'objet technique n'est alors plus une fin en soi, mais un moyen de faire sens dans une situation plus vaste (ici, raconter une histoire au moyen d'objets technologiques robotisés, dans un décor, une succession d'événements, etc.). On situe l'objet créé dans une pragmatique du sens, où le sens du signe se détermine en fonction de son usage, de son contexte et de sa réception par ceux qui le perçoivent.

La principale contrainte des défis proposés aux étudiant-e-s est d'adapter un texte littéraire au moyen de robots et d'en faire une petite pièce de théâtre. Le travail de transposition d'un texte qui induit l'intégration de robots, confrontera l'étudiant-e à différents problèmes. En relevant ces problèmes comme des éléments clefs lors de la construction de séquences d'enseignement-apprentissage, nous privilégions l'exploitation des situations problèmes. Le défi proposé n'est pas d'emblée de nature scientifique, car il convoque d'abord une réflexion sur le sens : quel sens voulons-nous donner à l'adaptation théâtrale ? Quel rôle auront les robots ? Pourquoi ? Que voulons-nous signifier avec la présence de robots dans la pièce ou générer chez les spectateurs (socialisation de l'objet) ? C'est en passant au niveau de la 
réalisation concrète du défi que l'on fait appel à l'esprit scientifique : le sujet se trouve confronté à des problèmes qui ne présentent pas une solution unique et dont certains composants sont incertains (Orange). Ce mécanisme spécifique à la représentation du problème et à sa modélisation nous renvoie à la résolution des problèmes de conception. Ceux-ci reprennent des spécificités de la typologie des problèmes en se caractérisant en tant que problèmes «mal définis », «astucieux» (wicked), «mixtes», « sémantiquement riches», « ouverts» ou «mal structurés » (Bonnardel 45). Les problèmes de conception qualifiés de wicked problems (problèmes astucieux) se caractérisent par une formulation qui dépend de la conception du monde dont disposent les concepteurs et qui conduit à une solution (Rittel et Webber). La spécificité de ces problèmes se constitue en tant que problème mixte en associant les caractéristiques d'autres types de problèmes (Greeno). En cela, la résolution des problèmes de conception nécessite une très bonne connaissance spécifique du domaine en question. L'intérêt de structurer les actes de dévolution autour des problèmes de conception consiste donc à déployer des mécanismes faisant appel à la créativité à l'aide de la pensée divergente et de la pensée convergente. Le problème de conception qualifié de problème «ouvert », admettant plusieurs solutions, n'est ni prévisible ni fini (Fustier). Le fait de privilégier un enseignement construit autour de la résolution de problèmes, consolide l'implémentation de démarches de recherches en ingénierie. Ainsi, l'exploration des mécanismes des problèmes de conception renforce la question des apprentissages et l'accès à la métacognition du sujet amené à résoudre le problème. En se confrontant à différents problèmes de programmation ou de construction de robots ou des parties de robots, l'étudiant met en œuvre un processus créatif dans lequel il va créer et innover (car aucune solution ne préexiste pour le problème donné).

\section{Le dispositif de formation}

\subsection{Description du dispositif de formation}

L'activité proposée dans ce module consiste à adapter un album pour enfants de façon à le mettre en scène au moyen de robots. Les étudiant-e-s choisissent quelques moments de l'histoire qui pourront être joués par des robots. Ils réalisent ainsi une courte pièce de thêâtre (qu'ils peuvent filmer et monter s'ils le souhaitent). Les étudiants testent ainsi une activité qu'ils réaliseront ensuite avec leurs propres élèves en classe. Réaliser eux-mêmes les défis leur permet d'appréhender la complexité de la tâche, mais aussi de s'approprier les différents champs disciplinaires requis par l'activité (connaissances techniques de l'usage et de la construction des robots, connaissances de programmation, de mécanique, champs du français, passage d'un texte écrit à son oralisation, apprentissage de la prise de vue, du montage vidéo, 
etc.). Les étudiant-e-s ont le choix entre différents types de robots. L'utilisation de robots déjà construits (comme la Bee-bot, le Thymio ${ }^{3}$ ), ou de machines à concevoir soi-même (par exemple avec les kits lego We-Do ou Mindstorms) facilite l'apparition d'idées nouvelles et adaptées au contexte. Les étudiant-e-s doivent également concevoir les décors et l'environnement sonore de la pièce (musique, bruitage, voix). Le travail est réalisé par groupes de trois à quatre étudiant-e-s. Le module a lieu dans une salle d'activités créatrices et manuelles, équipée d'ordinateurs portables, de caméras numériques et de wifi. Il est donc possible d'y travailler autant le bois, le carton, le papier que les supports numériques.

Pour revenir sur le déroulement de l'activité, le cours est composé de modules de quatre heures, ce qui permet d'associer dans une même séance et un même lieu, réflexion théorique et réalisations pratiques. Chaque groupe décide de son mode d'organisation. L'activité étant mise en place sur le mode de la pédagogie de projet, il s'agit également de faire prendre conscience aux futurs enseignant-e-s des éléments nécessaires à la mise en place collective d'un projet complexe sur une durée relativement longue (quatre mois). Les étudiant-e-s ont donc dû se répartir les tâches, autant pour les séances en atelier que pour celles à réaliser en dehors du cours. Certains ont sollicité la contribution de leurs élèves dans leurs classes de stage (réalisation de décors). Les formateurs étaient présents lors des séminaires, comme personnesressources.

\subsection{Transposition d'un texte en spectacle}

L'idée d'intégrer le robot dans un spectacle prend sa source dans un objectif de réflexion sur la socialisation de l'objet réalisé. Quel sens va prendre l'objet construit ? Comment sera-t-il utilisé et dans quel contexte ? Les textes choisis par les étudiants devaient permettre la mise en scène de plusieurs scénettes incluant des robots, soit comme personnages ou éléments du décor. Il s'agissait là d'une première contrainte et non des moindres. En effet, imaginer qu'un personnage soit incarné par un robot, ou qu'un mouvement du décor par exemple soit actionné par un robot demande de passer plusieurs niveaux de transposition.

Tout d'abord, comme dans une adaptation théâtrale, on passe d'un texte fait pour être lu, à un texte qui sera énoncé ou encore représenté de façon non-verbale, par des images, gestes, bruitages, musiques, objets, lumières, etc. Nous passons donc d'un mode de signification unique (un même type de signes, par exemple seulement du texte) à une pluralité possible de signes (images, textes, signes graphiques, sonores). Les étudiant-e-s produisent ainsi à partir du texte un scénario, dessiné sous forme de story board. Ils s'approprient ainsi une nouvelle forme de communication, qui doit aussi bien tenir compte de ce qui se montre, que de ce qui se dit. Durant la représentation interviennent autant les gestes et les mouvements que l'espace, 
les couleurs, les formes et les sons. La première étape de transposition consistait à extraire du texte initial des moments-clés de l'histoire, où les actions ou événements sont particulièrement propices à l'utilisation d'une machine. Quelques-uns des moments choisis, par exemple pour le chaperon rouge : la traversée de la forêt et la dévoration du chaperon par le loup. Le robot a aussi bien été utilisé pour représenter certains personnages (le loup par exemple), ou leur permettre de se déplacer (par exemple sur un char, une monture), ou encore de se déplacer dans les airs, en utilisant une poulie actionnée par un moteur.

\subsection{Conception des robots et des projets}

L'association entre le français, les activités créatrices et manuelles et les Mitics, nous amène à valoriser un enseignement par projet très proche de la formation des ingénieurs. De ce fait, nous partons du constat de Michel Sonntag : «L'enseignement de la conception ou la formation à la conception ont toujours été au cœur de la formation des ingénieurs pour qu'ils soient capables de mettre en œuvre leurs connaissances scientifiques et technologiques pour résoudre des problèmes techniques et industriels » (3). Pourtant, la recherche en didactique focalisée sur l'exploitation de la conception dans l'enseignement obligatoire est un domaine relativement nouveau, car réservé essentiellement à la formation professionnelle. Cet objet de recherche disciplinaire trouve pourtant des fondements dans l'approche anthropologique de Claude Lévi-Strauss. En effet, celui-ci compare les démarches du bricoleur, de l'ingénieur, du savant et de l'artiste qu'il situe de manière singulière et spécifique lors de leur action sur la matière. Cette approche anthropologique de l'activité se caractérise en tant que fondement didactique en activités créatrices permettant de cerner les démarches spécifiques de conception aux modes d'action sur la matière. Ce faisant, nous invitons les enseignant-e-s en formation à concevoir des séquences à l'aide de robots dans lesquels les élèves seront amenés à investir ces démarches. La conception de sculptures animées à l'aide de robots ou de machines à fonctions utilitaires invite rapidement les étudiant-e-s à quitter une posture d'exécutant pour être tour à tour confrontés à une démarche d'artiste, de bricoleur, d'ingénieur ou de scientifique. Dans les différents cas, nous privilégions de la part des étudiant-e-s l'appropriation d'une posture d'auteur (Dumas) de sa séquence d'enseignement. Celui-ci doit-être capable de partir d'une histoire et de la transposer sous forme de scénarios intégrant des robots. La notion d'auteur va donc être travaillée sous forme de réécriture d'une histoire initiale en une nouvelle histoire focalisée sur l'intégration de robots ou d'automates. En parallèle de la réécriture de l'histoire, les étudiant-e-s ont donc dû imaginer des petits robots qui seront intégrés dans la pièce. Les différents groupes ont procédé pour cette réalisation de différentes manières. Certains ont dessiné des plans, en passant immédiatement à un niveau d'abstraction assez élevé. D’autres 
groupes se sont immédiatement lancés dans la construction d'objets, par exemple de marionnettes articulées (pour ensuite y ajouter des mouvements robotisés).

Les groupes qui se sont plongés immédiatement dans de la réalisation (décors, robots), ont pu voir immédiatement les limites des outils et des matériaux à disposition (parfois trop lourds pour les mouvements voulus, moteurs pas assez puissants pour soulever ou déplacer les personnages, etc.), ils ont également rapidement testé les possibilités de programmation. Ces tests et confrontations avec les possibles les ont parfois conduits à repenser la forme du robot, voire même ses mouvements et déplacements. En quelque sorte les contraintes pratiques ont amené les étudiant-e-s à faire preuve de pensée divergente. La solution imaginée, souvent en apparence la plus simple, ne fonctionnant pas toujours, il a fallu trouver d'autres solutions, contourner les difficultés, voire inventer d'autres modes de représentation. Mais en commençant immédiatement par la construction et la pratique, ils ont parfois perdu de vue l'objectif final de leur projet, à savoir les significations qu'ils voulaient transmettre aux spectateurs de leur pièce, les expériences ou émotions qu'ils souhaitaient leur faire vivre. D'autres groupes d'étudiant-e-s ont privilégié une approche plus abstraite, en concevant au préalable l'ensemble de l'activité sous forme de schémas et de scénarios. Ces groupes se sont confrontés à d'autres difficultés, qui les ont notamment aussi contraints à parfois revoir toute la scénographie imaginée, car une fois le story board réalisé, ils se sont rendu compte d'impossibilités pratiques ou techniques. Ce type de problèmes aurait pu être évité avec une bonne connaissance préalable du champ de la robotique, mais la plupart de ces étudiant-e-s découvraient ce champ presque en même temps qu'ils débutaient leur projet.

Ce travail d'adaptation et de transposition d'un texte dans une scène animée et visuelle vise à tester différents types de signifiants que l'on peut employer dans la communication. Dans les différents projets, les étudiant-e-s ont, à ce sujet, souvent modifié leurs plans initiaux, au vu du résultat et de l'effet produit. Ils ont ainsi expérimenté une pragmatique de la communication, sur le mode de l'essai-erreur. Tel bruitage, éclairage, mouvement, produit-il l'effet voulu ? Ils ont également dû s'interroger sur le sens de l'usage d'une machine dans telle ou telle scène. Veut-on montrer le robot en tant que tel ? Ou dissimuler le mécanisme pour produire un effet, une illusion ? Qu'est-ce qui est mis en scène, la machine elle-même ou seulement le personnage, ou encore provoque-t-elle une ambiguïté sur la nature même du personnage (animal-robot) ?

Lorsque la pièce fut réalisée (avec des marionnettes, robots et autres objets), le passage à la réalisation d'un petit film a posé de nouvelles contraintes. En effet, la captation de la scène par la caméra ne correspondait pas toujours à la vision à l'œil nu et rendait impossible la 
perception de certains éléments (perspective, vue des décors). Mais par ailleurs, le mouvement possible de la caméra (zoom, déplacements, etc.) et les ajouts en post production (sons, bruitages, musiques, effets) rajoutent d'autres modes d'expression qui peuvent enrichir le produit final. Les étudiants découvrent alors que la réalisation du film n'est pas une simple captation visuelle d'un événement théâtral, mais un autre langage, avec d'autres possibilités et limites. Le montage notamment et le recadrage permettent de mettre en avant certains éléments ou d'en dissimuler d'autres, voire même de créer des illusions.

\section{Conclusion}

Dans cet article, nous avons souhaité montrer comment l'introduction d'une créativité appliquée par l'implémentation de robots peut favoriser la conception de séquences créatives en travaillant l'interdisciplinarité de manière innovante. La conception des séquences innovantes nécessite plusieurs transpositions successives, du texte à la représentation théâtrale (en quatre dimensions, espace et temps), puis à partir du théâtre, on retourne à une représentation en trois dimensions avec l'image filmique (image en 2D et temps). La technologie intervient ainsi à trois moments-clés de l'activité, d'une part en tant que conception et design dans la réalisation du robot, puis dans la programmation et, dans un troisième temps, dans la réalisation du film (prise de vue, montage, sonorisation). L'intérêt de ces activités consiste à mettre en place des projets à plusieurs facettes qui intègrent la technologie dans une perspective culturelle ou communicationnelle en s'irriguant par des activités enrichies d'objectifs d'apprentissage scientifiques et techniques. Ceci favorise l'acquisition de compétences communicationnelles (pragmatique, langages symboliques). Le travail sur l'objet technique fait ainsi partie de la création de moyens d'expressions. La technique devient alors un moyen de communiquer, parmi d'autres, combiné avec d'autres pratiques (culturelles, langagières). Ainsi, nous développons chez nos étudiants, l'apprentissage et l'appropriation d'outils techniques ou technologiques de manière créative. Certaines formes de pédagogie (par projet, par résolution de problèmes) se montrent particulièrement fécondes pour l'implémentation d'outils technologiques. La créativité se voit donc développée dans une approche interdisciplinaire où la motivation, l'originalité, l'imaginaire et le défi participent à l'augmentation de la prise de risques chez les étudiant-e-s tout en les habituant à innover dans leur capacité à concevoir des séquences nouvelles et adaptées aux élèves. 


\section{Références bibliographiques}

Bonnardel, Nathalie. Créativité et conception. Approches cognitives et ergonomiques. Marseille : Solal, 2006.

Csikszentmihalyi, Mihaly. Creativity : Flow and the Psychology of Discovery and Invention. New-York : Harper Colins, 1996.

Dagognet, François. Eloge de l'objet. Mayenne : Vrin, 1989.

Deforges, Yves. L'ouvre et le produit. Seyssel : Champ Vallon, 1990.

---. De l'éducation technologique à la culture technique. Paris : ESF, 1993.

Didier, John et Denis Leuba. «La conception d'un objet : un acte créatif ». Prismes 15 (2011) : 32-33.

--- et Florence Quinche. « Robotique pédagogique ». Jeunes et médias 5 (2013) : 109-116.

Dumas, Gilbert. «Questions orales à Guy Brousseau dans Salin ». Sur la théorie des situations didactiques. Dir. Marie Hélène Clanché et Pierre Sarrazy. Grenoble : Pensée sauvage, 2005. 34-38.

Fustier, Michel. La résolution de problème: méthodologie de l'action. Paris : ESF \& Techniques, 1989.

Greeno, James G. « Natures of Problem-Solving Habilities ». Handbook of Learning and Cognitive Processes. Dir. W. K. Estes. Hillsdale, N.J. : Lawrence Erlaubm, 1978. 239270.

Guilford, Joy Paul. The Nature of Human Intelligence. New York : McGraw Hill, 1967.

Lamard, Pierre et Yves-Claude Lequin. La technologie entre à l'Université Compiègne, Sevenans, Belfort-Montbéliard. Belfort : U de Technologie de Belfort-Montbéliard, 2005.

Lévi-Strauss, Claude. La pensée sauvage. Paris : Plon, 1962.

Lubart, Todd. Psychologie de la créativité. Paris : Colin, 2003.

Orange, Christian. «Problème et problématisation ». Aster 40 (2005) : 3-11.

Quinche, Florence et John Didier. «Développer la créativité des élèves au moyen de la robotique ». Educateur 2 (2014) : 11-12.

---. «Les apports de la robotique aux apprentissages ». Prismes 16 (2012) : 53-54.

Rittel, Horst et Melvin M. Webber. «Planning Problems are Wicked Problems». Developments in Design Metholodology. Dir. Nigel Cross. New-York : Wiley. 1984. 134-135.

Simondon, Gilbert. L'individuation psychique et collective. Breteuil sur Iton : Aubier, 1989.

Sonntag, Michel. «La conception au cœur de la formation professionnelle ». Les sciences de 
l'éducation - Pour l'ère nouvelle 40 (2007) : 59-78.

Terssac, Gilbert, de et Erhard Friedberg. Coopération et conception. Toulouse : Octarès, 2002.

Torrance, Ellis Paul. Tests de pensée créative. Paris : Centre de Psychologie Appliquée, 1976.

\begin{abstract}
NOTES
${ }^{1}$ Ce type de problème s'inspire des méthodes d'apprentissage par problème en mathématiques et en physique, où la réponse attendue est déjà connue de l'enseignant et où une seule réponse juste est acceptable.

${ }^{2}$ «Le modèle théorique Conception-Réalisation-Socialisation se conçoit comme une cartographie de l'activité développée dans son ensemble et dans sa complexité lors de la fabrication d'un objet. Les fondements de ce cadre théorique sont multiples et puisent leur articulation sur trois temporalités distinctes, la conception, la réalisation et la socialisation »(Didier et Leuba)

3 Robot réalisé par l'école polytechnique de Lausanne (EPFL), comportant de nombreux capteurs et ayant plusieurs comportements pré-enregistrés, il peut être utilisé en mode tactile par des élèves des 4-5 ans ou à partir de $8-10$ ans avec le mode de programmation graphique. https://aseba.wikidot.com/fr:thymio
\end{abstract}

